\title{
Searches for direct top squark pair production in compressed Supersymmetry scenarios with the ATLAS detector
}

\author{
Samuel David Jones* on behalf of the ATLAS Collaboration \\ University of Sussex \\ E-mail: samuel.david.jones@cern.ch
}

\begin{abstract}
If Supersymmetry is to stabilise the Higgs boson mass, naturalness arguments suggest the mass of the scalar partner of the top quark (the stop) to be below around $1 \mathrm{TeV}$. The decay of the stop depends in part on the mass difference $\Delta m=m_{\tilde{t}}-m_{\tilde{\chi}_{1}^{0}}$ between the stop and the lightest neutralino, assumed to be stable. For $\Delta m<m_{W}+m_{b}$ the process $\tilde{t} \rightarrow c+\tilde{\chi}_{1}^{0}$ may dominate. This contribution presents a summary of stop searches targeting this region of parameter space. Conducted using a newly developed tagger for the identification of charm quarks, and using 36.5 $\mathrm{fb}^{-1}$ of proton-proton collisions collected by the ATLAS detector at a centre-of-mass energy of $\sqrt{s}=13 \mathrm{TeV}$, these searches significantly extend the exclusion limits in the $\left(m_{\tilde{\chi}_{1}^{0}}, m_{\tilde{t}}\right)$ parameter space made by ATLAS during Run 1 of the LHC.
\end{abstract}

The European Physical Society Conference on High Energy Physics

5-12 July, 2017

Venice

${ }^{*}$ Speaker. 


\section{Introduction}

Supersymmetry (SUSY) is an appealing extension to the Standard Model (SM) that could stabilise the Higgs mass and provide a candidate for Dark Matter in the Universe. In generic Rparity conserving Minimal Supersymmetric Standard Model (MSSM) theories, SUSY particles are pair produced and the lightest supersymmetric particle (LSP) is stable.

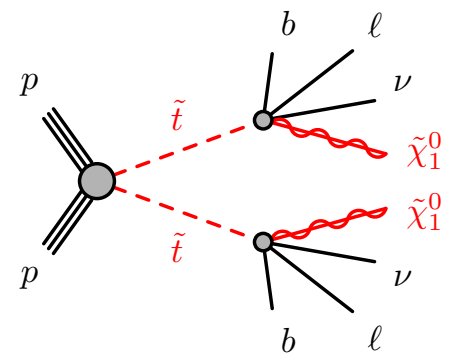

(a)

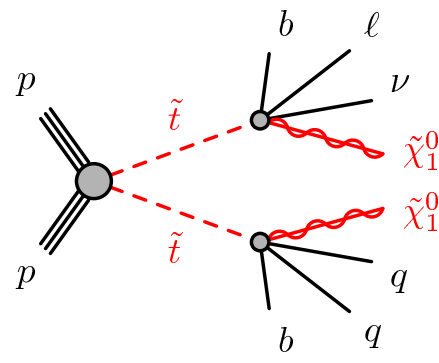

(b)

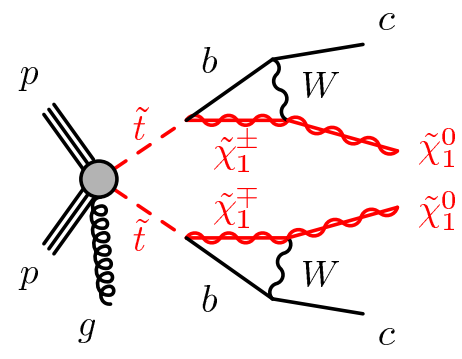

(c)

Figure 1: Illustration of the SUSY signatures considered for these searches.

Naturalness arguments suggest the mass of the scalar top (stop) to be below around $1 \mathrm{TeV}$, accessible to the LHC. This proceedings contribution presents searches for direct stop pair production at ATLAS [1] in compressed regimes, where the stop is assumed to decay to the LSP, $\tilde{\chi}_{1}^{0}$, and the mass difference $\Delta m=m_{\tilde{t}}-m_{\tilde{\chi}_{1}^{0}}$ is small, which is an interesting region of phase space since it can satisfy the dark matter relic density through stop-neutralino coannihilation [2].

\section{Search Strategies}

Signal regions (SRs) are obtained by applying a series of cuts on kinematic variables designed to target a particular signal and eliminate background processes. Data driven estimates for irreducible backgrounds are obtained by designing control regions (CRs), orthogonal to the SRs, that are rich in a particular background and low in signal. The extrapolation from the CRs to the SRs is validated using dedicated validation regions (VRs) that are somewhere between the SRs and CRs. Each of the searches outlined below uses an $E_{\mathrm{T}}^{\text {miss }}$ based trigger to select data events and exploit high $E_{\mathrm{T}}^{\mathrm{miss}}$ from the LSP and high leading jet $p_{\mathrm{T}}$ from the stop decay.

\section{1 $\tilde{t} \rightarrow b f f^{\prime} \tilde{\chi}_{1}^{0} 2$ Lepton Signal}

This signature, shown in figure 1(a), is characterised by the presence two opposite sign leptons in the final state. The SR requires these to be reconstructed and applies a mass cut to reject low mass resonances. The ratio variables:

$$
R_{l l}=E_{\mathrm{T}}^{\mathrm{miss}} /\left(p_{\mathrm{T}}\left(\ell_{1}\right)+p_{\mathrm{T}}\left(\ell_{2}\right)\right)
$$

and

$$
R_{l j}=E_{\mathrm{T}}^{\mathrm{miss}} /\left(E_{\mathrm{T}}^{\mathrm{miss}}+p_{\mathrm{T}}\left(\ell_{1}\right)+p_{\mathrm{T}}\left(\ell_{2}\right)+\sum_{i=1, \ldots, N \leq 4} p_{\mathrm{T}}\left(j_{i}\right)\right)
$$

are used to reject background events, which peak at lower values [3]. 


\section{2 $\tilde{t} \rightarrow b f f^{\prime} \tilde{\chi}_{1}^{0} 1$ Lepton Signal}

This signature, shown in figure 1(b), is targeted by a dedicated soft-lepton selection, where the lepton $p_{\mathrm{T}}$ threshold is relaxed to target the low $\Delta m$ signal. It is characterised by the presence of two jets from bottom quarks, which are identified by a dedicated tagger, based on a boosted-decisiontree (BDT) multivariate analysis (MVA) method. $t \bar{t}$ is a dominant background in this analysis; it is reduced by applying a $\chi^{2}$ based top tagger [4].

\section{$2.3 \tilde{t} \rightarrow c \tilde{\chi}_{1}^{0}$ O Lepton Signal}

A key discriminating feature is the presence of two charm jets in the final state (see figure 1(c)). A newly developed tagger based on a variant of the BDT based $b$-tagger, trained on MC samples of bottom, charm and light flavoured jets, is deployed to identify these jets. In the more compressed scenarios, closer to line $m_{\tilde{t}}=m_{\tilde{\chi}_{1}^{0}}$, the physics objects in the final state are too soft to be reconstructed, and the presence of an ISR jet is required to boost the system. Important kinematic variables for this search are $E_{\mathrm{T}}^{\text {miss }}$, leading jet $p_{\mathrm{T}}$ and the variable

$$
m_{\mathrm{T}}^{c}=\min _{j \in c \text {-tagged jets }} \sqrt{2 E_{\mathrm{T}}^{\mathrm{miss}} E_{\mathrm{T}}^{c_{j}}\left[1-\cos \Delta \phi\left(\vec{p}_{\mathrm{T}}^{\mathrm{miss}}, \vec{p}_{\mathrm{T}}^{c_{j}}\right)\right]}
$$

designed to reject $W \rightarrow \tau \nu$ with a hadronically decaying $\tau$ mistagged as a $c$-jet. The main irreducible backgrounds for this analysis are $Z \rightarrow v v$ and $W \rightarrow l v$ with associated jets, and $t \bar{t}$. Multijet QCD background events can enter the SRs due to mismeasured $E_{\mathrm{T}}^{\text {miss }}$, resulting in jets aligned with $\vec{E}_{\mathrm{T}}^{\text {miss }}$. These are rejected by imposing a cut on $\min _{j \in \text { jets }} \Delta \phi\left(\vec{E}_{\mathrm{T}}^{\text {miss }}, p_{\mathrm{T} j}\right)$. The irreducible backgrounds are constrained in 1 lepton and 2 lepton CRs.

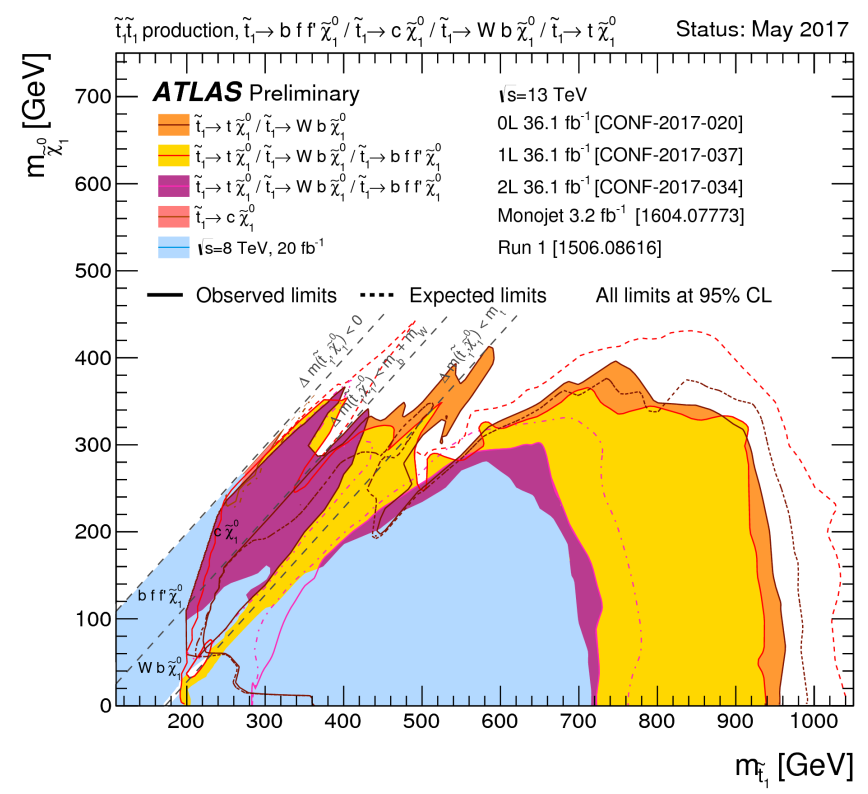

Figure 2: Current exclusion limits in the $\left(m_{\tilde{t}}, m_{\tilde{\chi}_{1}^{0}}\right)$ plane [6]. 


\section{Results and Outlook}

The results for the $1 \mathrm{~L}$ and $2 \mathrm{~L}$ searches have been made public. No significant excess over SM predictions was observed. The null result was used to place exclusion limits in the $\left(m_{\tilde{t}}, m_{\tilde{\chi}_{1}^{0}}\right)$ plane, shown in figure 2 . The Run 1 limits are significantly extended by these results.

The $\tilde{t} \rightarrow c \tilde{\chi}_{1}^{0}$ results were obtained from Run 1 and it is anticipated that Run 2 results will extend the limits in the compressed regime, close to the diagonal. ATLAS is now collecting data and these searches will benefit from increased integrated luminosity, as well as from ongoing research and developement, to increase the limits further and enhance the chance of discovery.

\section{References}

[1] ATLAS Collaboration, The ATLAS Experiment at the CERN Large Hadron Collider, 2008 JINST 3 S08003

[2] M. Adeel Ajaib, Tong Li, Qaisar Shafi, Stop-Neutralino Coannihilation in the Light of LHC, 2011 arXiv:1111.4467

[3] ATLAS Collaboration, Search for direct top squark pair production in final states with two leptons in $\sqrt{s}=13$ TeV pp collisions with the ATLAS detector, ATLAS-CONF-2017-034, 2017 https://cds.cern.ch/record/2265806

[4] ATLAS Collaboration, Search for top squarks in final states with one isolated lepton, jets, and missing transverse momentum using $36.1 \mathrm{fb}^{-1}$ of $\sqrt{13} \mathrm{TeV}$ pp collision data with the ATLAS detector, ATLAS-CONF-2017-037, 2017 https://cds.cern.ch/record/226617

[5] ATLAS Collaboration, Search for pair-produced third-generation squarks decaying via charm quarks or in compressed supersymmetric scenarios in pp collisions at $\sqrt{13} \mathrm{TeV}$ with the ATLAS detector, 2014 arXiv:1407.0608

[6] The ATLAS Collaboration Supersymmetry Public Results https://twiki.cern.ch/twiki/bin/view/AtlasPublic/SupersymmetryPublicResults 